\title{
Associations between patient experience and clinical outcomes at a level I trauma center: a cross-sectional survey-based study
}

\author{
Andrew Oberle, Dajun Tian, Kyle Hayes, Steven Howard, Vicki Moran \\ Saint Louis University, St. Louis, MO, USA \\ Contributions: (I) Conception and design: All authors; (II) Administrative support: A Oberle, V Moran; (III) Provision of study materials or patients: \\ A Oberle, V Moran; (IV) Collection and assembly of data: A Oberle, D Tian, K Hayes, V Moran; (V) Data analysis and interpretation: D Tian, K \\ Hayes, A Oberle, S Howard; (VI) Manuscript writing: All authors; (VII) Final approval of manuscript: All authors. \\ Correspondence to: Andrew Oberle, MHA, MA. Executive Director-Oberle Institute, School of Medicine; PhD Student in Public Health Studies/ \\ Health Management and Policy; College for Public Health and Social Justice, Saint Louis University, SLUCare Academic Pavilion, 1008 S Spring \\ Ave., St. Louis, MO 63110, USA. Email: andrew.oberle@health.slu.edu.
}

Background: Helping survivors of traumatic injuries achieve optimal recovery is a crucial global health issue. Traumatic injuries present major implications for the health of patients, care systems, and world economies. Focusing on the improvement of structures and processes that influence hospitalized trauma patient experience is a potentially important way to improve outcomes and health-related finances. The purpose of this study is to determine if there are associations between patient experience and clinical outcomes for hospitalized trauma patients.

Methods: The study used a cross-sectional design. Primary data was collected in March and April 2020. Potential participants were 95 trauma patients who received care for traumatic injuries at a United States urban Level I trauma center from November 2018 to January 2020 and consented to be contacted for future research. Phone surveys were used to collect quantitative and qualitative data on participants' patient experiences and health outcomes related to their hospitalization. Additional health outcomes were collected from participants' electronic health records. General linear and Poisson regressions were used to analyze associations between experience and outcomes. Differences between injury severity groups were analyzed using chi-square and t-tests.

Results: Thirty participants completed the phone survey, a response rate of $31.6 \%$. Positive nurse communication was associated with a significantly lower risk of hospital-acquired complications compared to negative nurse communication [ $-33 \%$; $95 \%$ confidence interval (CI), $-61 \%$ to $-5.5 \%$. Patients with severe injuries had a lower risk of 30-day readmissions when reporting positive nurse communication $(-56 \%$ decrease; $95 \% \mathrm{CI},-88 \%$ to $-23 \%)$, positive doctor communication (-50\%; $95 \% \mathrm{CI},-81 \%$ to $-19 \%)$, and positive overall hospital rating $(-56 \%$; $95 \%$ CI, $-99 \%$ to $-13 \%)$ when compared to severely injured patients who reported negative nurse and doctor communication and overall rating.

Conclusions: This study shows that aspects of patient experience, especially those related to communication with providers, are significantly associated with clinical outcomes for acute trauma patients, with potential implications for Continuous Quality Improvement and value-based reimbursement. Additional research would confirm if these associations exist for larger samples and patients treated for traumatic injuries in non-urban settings.

Keywords: Patient experience; quality of care; trauma care

Received: 14 September 2021; Accepted: 14 January 2022; Published: 25 September 2022.

doi: 10.21037/jhmhp-21-65

View this article at: https://dx.doi.org/10.21037/jhmhp-21-65 


\section{Introduction}

Helping trauma survivors achieve the best possible recovery is a crucial global health issue because traumatic injury is the third leading cause of death and disability-adjusted life years (DALYs) (1). Over one-quarter of these trauma survivors sustain debilitating injuries that can lead to poor long-term health outcomes, chronic physical and mental health issues, and loss of employment and other productive and meaningful activity (2). While continuously improving the patient experience for this population is important for health care finance, ensuring optimal patient experience can also have a major impact on an individual's successful recovery (3-5). According to the Donabedian conceptual model (6), which is used to examine and evaluate the structures, processes, and outcomes that influence care quality, better trauma care structures and processes should lead to improved patient outcomes $(7,8)$. However, the associations between trauma patient experience and outcomes are inconsistent (9-11). Furthermore, traditional representations of trauma care processes limit the importance of patient experience and don't take patientcentered care (PCC) into account $(12,13)$. The purpose of this study is to determine if patient experience is associated with important clinical outcomes in an urban level I trauma center in the United States. Understanding whether enhancing patient experience affects outcomes, such as 30day readmissions and hospital acquired complications, is important for both the patient and the trauma health care system. If strong associations exist, trauma care leaders may be able to enhance the recovery of their patients and the financial sustainability of the care they provide by improving patient experience.

\section{Trauma patient experience}

Ensuring trauma patients achieve the best possible outcomes is increasingly important as the prevalence of traumatic injuries around the world has contributed to a global public health crisis that affects both the health and economies of nations $(14,15)$. Vos and colleagues estimated that around 1.83 billion people were treated for traumatic injuries in 2019, which led to 249 million DALYs (1), a measure of population health loss that sums the years of life lost due to premature mortality and years lived with a disability (16). For individuals aged 10 to 49, road injuries are the leading cause of DALYs and males are disproportionately affected (1). As of 2010, 5.8\%
(56.2 million) of people injured around the world required acute care hospitalization for injury treatment (2). In the United States (US), traumatic injuries account for approximately 2.5 million hospital admissions per year (17) and close to $26 \%$ of those hospitalized sustained severe injuries that can lead to disability, reduced quality of life, and unemployment $(3,15)$. These debilitating injuries account for approximately $30 \%$ of all life years lost (more than cancer, heart disease, and HIV combined), and US\$396 billion in annual healthcare costs and lost productivity (18).

The Agency for Healthcare Research and Quality (AHRQ) defines patient experience as the "range of interactions that patients have with the health care system, including their care from health plans, and from doctors, nurses, and staff in hospitals, physician practices, and other health care facilities (19)." Focusing on patient experience is important for care delivery and payment throughout the world, including the United States (20), United Kingdom (21-24), the Netherlands (25,26), Germany (27-29), Denmark (30), and Canada (31). Furthermore, the Organization for Economic Co-operation and Development (OECD) recommends that all participating countries measure and address patient experience as part of health quality improvement initiatives (32) and the Commonwealth Fund supports and conducts patient experience research throughout Europe and the US to better understand the perspectives of patients and achieve their mission of improving health care for all (33).

In the US., the Institute of Medicine's 2001 "Crossing the Quality Chasm" report, emphasized the first fundamental change necessary for improving quality is centering care on the experience of patients, families, and their communities $(12,34,35)$. The Hospital Consumer Assessment of Healthcare Providers and Systems (HCAHPS) (36) is used to adjust reimbursement through the Hospital ValueBased Purchasing Program (HVBP) further solidified this new focus by promoting and incentivizing new delivery methods, such as care integration and coordination under accountable care organizations (37). Patient-centered self-reported outcomes of experience, such as HCAHPS, have become increasingly important metrics for hospitals' financial stability and competitive ability because they represent individuals' evaluation of various aspects of their experience, including communication with providers and overall rating of the hospital. Hospitals may receive lower levels of reimbursement if HCAHPS scores are below the acceptable standard $(38,39)$.

Examining how subgroups of patients, such as those 
receiving acute trauma care, experience hospitalization is important because different populations may evaluate their experience in different ways. Aspects of patient experience that a traumatically injured individual finds important, such as pain management, may not be as important to someone recovering from a heart attack (40). Hospital trauma centers are crucial facilities for addressing issues that affect the recovery of injured patients because they are the point of entry into the health care system and facilitators of transition to post-acute care. However, providing acute care to this patient population is especially costly for hospitals and clinicians because trauma patients represent a large proportion of hospital admissions and care related costs, while the payer mix is often disproportionately composed of individuals of lower socioeconomic status (SES) who are either uninsured or rely on lower-paying reimbursement sources, such as Medicaid (4). Focusing on continuously improving the quality and experience of acute trauma care is paramount to ensuring patients achieve the best possible clinical and experiential outcomes so hospitals can optimize financial performance through operational effectiveness and incentive-based reimbursement programs (3-5).

Patient experience, as measured by HCAHPS, is commonly reported using the top-box methodology, which is the percentage of patients giving the most positive responses. Six composite measures (nurse communication, doctor communication, responsiveness of hospital staff, communication about medicines, discharge information, care transition), two individual measures (cleanliness, quietness), and two global measures (hospital rating, willingness to recommend hospital) are publicly reported through the Hospital Compare website $(41,42)$. These measures may be the best source of information hospitals have to assess quality of interactions between patients and providers and barriers or areas of dissatisfaction patients may face $(9,10,24,29,40,43)$. The scores for the two global measures carry significant weight, as they directly impact revenue (44). All domains measured by HCAHPS have significant impact on the hospital rating, although two domains, nurse communication and doctor communication, have a greater impact on the overall score $(36,40,45,46)$.

\section{Health issue factors}

While the HVBP has brought attention to patient experience regarding hospital reimbursement and reputation, it has also increased the focus on PCC because some studies have found that patient experience measures are linked to patient outcomes. Proponents of HCAHPS believe the system captures features of self-reported and clinical care that are most important to patients because patient experience is influenced by their expectations on care delivery $(10,11)$. On the other hand, opponents of HCAHPS-based reimbursement systems, such as some providers and administrators, believe patients care more about non-medical, concierge-like services and that patient experience cannot accurately capture surgical and clinical performance that is necessary for positive health outcomes $(9,11)$. This difference is especially prominent in trauma centers where care specialists possess an increased amount of control over care decision making processes and patients have less autonomy due to the emergent, time-sensitive nature of cases (9).

\section{Rationale}

Donabedian's structure-process-outcome quality of care model (DM) (6) provides a conceptual model to better understand how improvement initiatives to enhance patient experience for trauma patients can lead to improved outcomes. According to the DM structural characteristics of hospitals (doctor and nurse-to-patient ratio, hospital size, etc.) have a direct influence on their clinical processes (technical skill of providers, interpersonal communication, etc.), which in turn, influence patient outcomes (patient experience and clinical outcomes) (8). Moore and colleagues (7) have validated the utility of the DM for evaluating care quality in trauma centers, showing that trauma centers with higher structural performance scores, as indicated by quality indicators (QIs) developed by the American College of Surgeons (ACS) (ex. having an on-call radiologist), have higher process performance scores, also indicated by ACS QIs (ex. not reintubating a patient within 48 hours of extubation). These higher-performing trauma centers had better outcomes than trauma centers with lower structure and process performance scores. However, ACS QIs do not necessarily represent structures and processes that relate to patient experience as measured by HCAHPS.

As discussed in the 2001 Quality Chasm Report (35), patient experience is a crucial component of PCC, evaluation of health care effectiveness, and continuous quality improvement (CQI). Providers working together with patients and their loved ones to collaboratively design personalized care plans is one of the many aspects that represents the interpersonal interaction and communication at the foundation of PCC. According to the DM, having 
structures, such as a PCC culture and evaluation capability, that promote processes, such as engaging with patients in a respectful and compassionate manner, will lead to improvements in patients experience and outcomes (13). Rathert and colleagues (12) identified several PCC processes that reflect HCAHPS composite measures. HCAHPS's composite measures (communication with staff, discharge planning and education) relate almost exclusively to PCC processes (respect for patient preferences and information, education, communication), while the global ratings (overall rating, would recommend hospital) can be considered a combination of care structures and processes. However, it is unclear if high performing PCC structures and processes that lead to higher PCC patient-reported outcomes will also lead to improved clinical outcomes, such as the common clinical indicators for acute trauma patient outcomes of hospital complications, length of stay (LOS), and 30-day readmission rates $(7,8,47-50)$.

Understanding patient experience and how it relates to structures, processes, and observed clinical outcomes would help determine if there are meaningful associations between these measures and inform trauma department managers' approaches to improving specific aspects of patient experience (40). If certain aspects of patient experience, as measured by HCAHPS, are significantly associated with health outcomes, then managers would benefit by focusing human and financial resources on improving those PCC processes to improve patient outcomes.

\section{Study objectives}

In this study, we explore whether HCAHPS measures of patient experience, representative of DM/PCC processes, are associated with clinical outcomes for acute trauma patients at an urban Level I trauma center. Furthermore, we examine if there are any differences in associations based on injury severity. Limitations of existing studies exploring associations between patient experience and patient outcomes are that researchers sometimes use hospital-level data that are easily available but may not represent specific care units within the hospital, potentially missing important patient characteristics and introducing biases during analysis $(9,51)$. Other studies may use trauma patient-level data but use it to predict which factors (specific experience domains and/or clinical outcomes) affect overall patient satisfaction with the hospital (52). To our knowledge, no other study uses individual trauma patient-level data to explore whether specific HCAHPS domains (i.e., interpersonal interactions with providers, discharge planning, and overall ratings) impact clinical outcomes (i.e., 30-day readmissions, hospital-acquired complications, and LOS) differently for trauma patients overall and specifically for patients who are severely injured compared to those who are not. We present the following article in accordance with the STROBE reporting checklist (available at https://jhmhp.amegroups. com/article/view/10.21037/jhmhp-21-65/rc).

\section{Methods}

\section{Study design}

This study uses a cross-sectional design with the patient as the unit of analysis. Each participant completed a brief survey over the phone about their experience and outcomes as a recent acute trauma patient at an US urban level I trauma center that provides care to patients who are injured in the St. Louis, Missouri, metropolitan area, and surrounding rural areas in Missouri and Illinois. The study was conducted in accordance with the Declaration of Helsinki (as revised in 2013). The study was approved by the Saint Louis University Institutional Review Board (IRB No. 31075) and informed consent was given by all individuals before participating in the survey.

\section{Recruitment and sampling}

Potential participants were 95 patients admitted to this Level I trauma center between November 2018 and January 2020 for traumatic injuries who consented while hospitalized to be contacted for future research. A small pool of potential participants may lead to a small sample size, which can limit the interpretation of results (discussed further below). Participants were recruited and surveyed via phone in March and April of 2020 by authors AO and KH. Each participant was called three times over the course of a week using a staggered schedule (once in the morning, the afternoon, and evening) to account for individuals' differing schedules and availability. If there was no answer, researchers left a message to explain the purpose of the call, leave a call-back number, and state a researcher would call back. When someone other than the potential participant answered, researchers asked for an appropriate time to call back. If the potential participant answered, researchers described the study and asked for consent to begin the survey. 


\section{Measures}

Patient experience was measured using the standard questions from the HCAHPS survey. These were questions related to nurse communication (questions 1, 2, and 3), doctor communication (questions 5, 6, and 7), overall hospital rating (question 18), likelihood of recommending the hospital to others (question 19), and aspects of their care transition (questions 20, 21, and 22). Self-reported health status was collected by asking participants to rate their current overall health and mental/emotional health. Demographic characteristics, including age, sex, and race, were collected as part of this study's survey, and used as covariates during analysis. Injury severity scores (ISS), based on ACS classification of not severe (ISS $<16$ ) and severe (ISS $\geq 16$ ) (49), were collected from each participant's electronic health record (EHR) and used as a covariate. The ISS has been the most commonly used injury severity rating for several decades and is standard practice in trauma centers with ACS certifications (53). A list of survey questions and answers can be found in Appendix 1.

After being asked the standard HCAHPS items, questions related to health outcomes were surveyed and used as predictors. Participants were asked to recall whether their LOS was longer than they expected, if they were readmitted to emergency or acute care within 30 days after discharge, and if they developed one or more of the eight most common hospital-associated complications for trauma patients. Common complications include pneumonia, urinary tract infection, drug or alcohol withdrawal syndrome, thrombophlebitis, unplanned intubation, and unplanned return to ICU (49). An additional outcome, actual LOS, was collected from each participant's EHR.

\section{Statistical analysis}

To address non-response bias, participants and nonresponders were compared based on the key demographic and health-related characteristics of age, sex, race, LOS, and ISS. HCAHPS reliability and construct validity were demonstrated by Keller and colleagues (54) but have not been evaluated for a subset of the HCAHPS survey in a specific population of Level I trauma patients in the US. Cronbach's coefficient alpha was used to estimate the internal consistency reliability for each of the composite measures of nurse communication, doctor communication, care transition, and an overall set of items. Reliability of the measures is considered acceptable if the Cronbach's alpha is greater than or equal to 0.7 (55). Spearman correlation coefficient between the composite measures (nurse communication, doctor communication, and care transition) and the two global measures (overall hospital rating, recommendation of hospital) was used to measure the convergent validity of the composite measures (56). Discriminant validity was evaluated for each of the composite measures (nurse communication, doctor communication, and care transition) by measuring correlations of the measures with overall health and overall mental health, respectively (36). The expectation is the composite measures are significantly correlated with global measures and all measures are correlated with overall health and overall mental health.

Following standard linear mean scoring or top-box scoring, all individual measures were coded into continuous scales $(0-10)$ or binary scales $(0 / 1)$. Then at the patient level, the continuous scale of composite measures is the arithmetic average of the individual measures. The composite measures were rated as positive only when all the individual measures were most positive (i.e., with value of 1 in the binary scale) (57). The main patient group variable was participants' ISSs. The difference between not-severe and severe groups was compared using chi-square tests, $t$-tests, or Wilcoxon tests whenever applicable.

For the binary outcomes of being readmitted for emergency or acute care within 30 days of discharge, developing complications, and staying longer than expected, we used Poisson regression to test the difference in proportion of the outcomes (58) and incorporated robust error variance to get reliable estimates. All models included single independent variables and the associations with outcomes were tested for severe injury, nurse communication, doctor communication, care transition, hospital rating, recommending of hospital, overall health, and overall mental health. We further stratified the analysis by injury group (not-severe/severe). Multivariate analysis was not attempted due to the small sample size. Analysis was conducted using SAS 9.4 (59) Proc Genmod step and a $P$ value less than 0.05 was considered significant.

\section{Results}

\section{Sample size}

Of the 95 initial potential participants, 18 had phone numbers on file that were either disconnected or not-inservice. Additionally, 42 didn't answer a working number three times or were not available when the call was answered. Five patients answered but declined participation. 


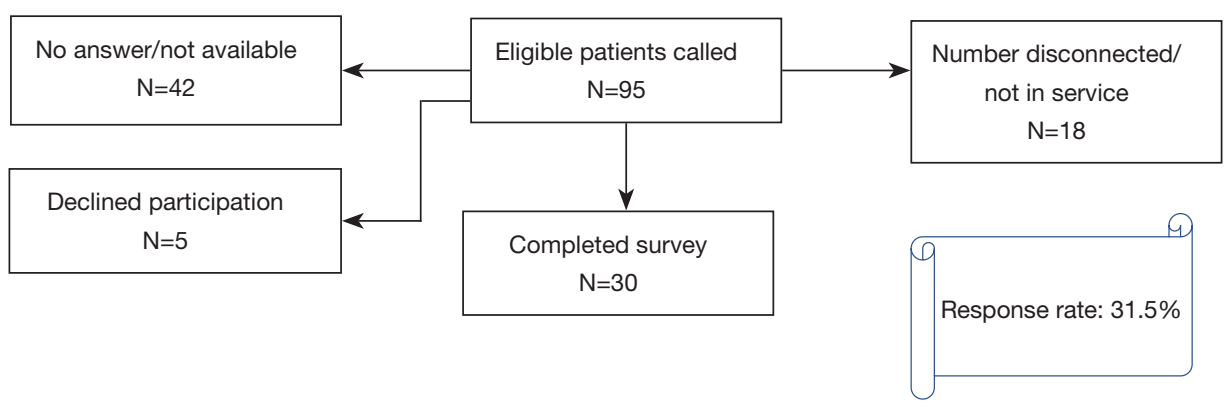

Figure 1 Participant flow diagram.

In total, 30 individuals completed the survey, a response rate of $31.5 \%$ (Figure 1 ).

\section{Descriptive analyses}

The average time between hospital discharge and survey for the sample was 11.87 (standard deviation $=3.55$ ) months, ranging from 3.87 to 16.07 months. Table 1 provides a demographic description of participants and comparisons by ISS. Additional race/ethnicity choices were included as a survey option, but all respondents fell into either White or Black/African American categories. There were no significant demographic differences between the severely injured and not-severely injured groups.

In this sample, 20 participants (67\%) gave the hospital an overall positive rating and 24 participants $(80 \%)$ would "definitely" recommend the hospital to their friends or family. Half of the participants $(\mathrm{n}=15)$ experienced positive nurse communication (giving the highest scores on all 3 questions) and positive care transition. Doctor communication scores were slightly lower, with 11 (37\%) participants considering their interactions positive. Only 3 (10\%) participants considered their overall health excellent, while 7 (23\%) reported excellent overall mental health. In total, 7 (23\%) participants had at least one readmission within 30 days of discharge and had LOSs longer than they expected. Only 4 (13\%) participants experienced hospitalacquired complications. The mean LOS for all participants was 8.2 days ( \pm 6.7 days) (Table 2). There were no significant differences in these measures by severity group and none of the participants were missing data.

\section{Reliability and validity}

Internal consistency reliability of the study's patient experience measures for this specific acute trauma patient sample was achieved for the overall set of patient experience measures (Cronbach's Alpha $=0.80$ ), nurse communication (Cronbach's Alpha $=0.74)$, and doctor communication (Cronbach's Alpha $=0.74$ ). Validity of the study's patient experience measures for this specific acute trauma patient sample was partially achieved as the Spearman's correlation coefficient was above 0.4 for pairwise correlation between doctor communication and recommendation; care transition and recommendation; and overall hospital rating and recommendation. As for discriminant validity, all items were weakly correlated $(r<0.2)$ with overall health or overall mental health, except between care transition and overall mental health $(\mathrm{r}=0.37)$.

\section{Associations between patient experience and outcomes}

We identified one significant association for the entire sample between patient experience and the outcome of hospital-acquired complications (HACs). We observed positive nurse communication (the risk factor) was associated with a significantly lower risk of HACs (the outcome) $[6.7 \%$ among participants with positive nurse communications experienced HACs compared to $40 \%$ among participants with negative nurse communications; difference: $-33 \%, \mathrm{P}=0.02,95 \%$ confidence interval $(\mathrm{CI})$, $-61 \%$ to $-5.5 \%$ ] (Figure 2).

In the subgroup analysis stratified by injury severity, we did not observe any significant correlations between patient experience ratings and outcomes in the not severely injured group (ISS $<16$ ). On the other hand, the severely injured group (ISS $\geq 16$ ) experienced significantly lower rates of 30-day readmissions when participants reported positive nurse communication (56\% decrease; $95 \% \mathrm{CI},-88 \%$ to $-23 \%)$, positive doctor communication (50\% decrease; $95 \%$ CI, $-81 \%$ to $-19 \%)$, positive overall hospital rating $(56 \%$ decrease; $95 \% \mathrm{CI},-99 \%$ to $-13 \%$ ), and that they would 
Table 1 Characteristics of level I trauma patients in the sample and univariate comparison by injury severity score

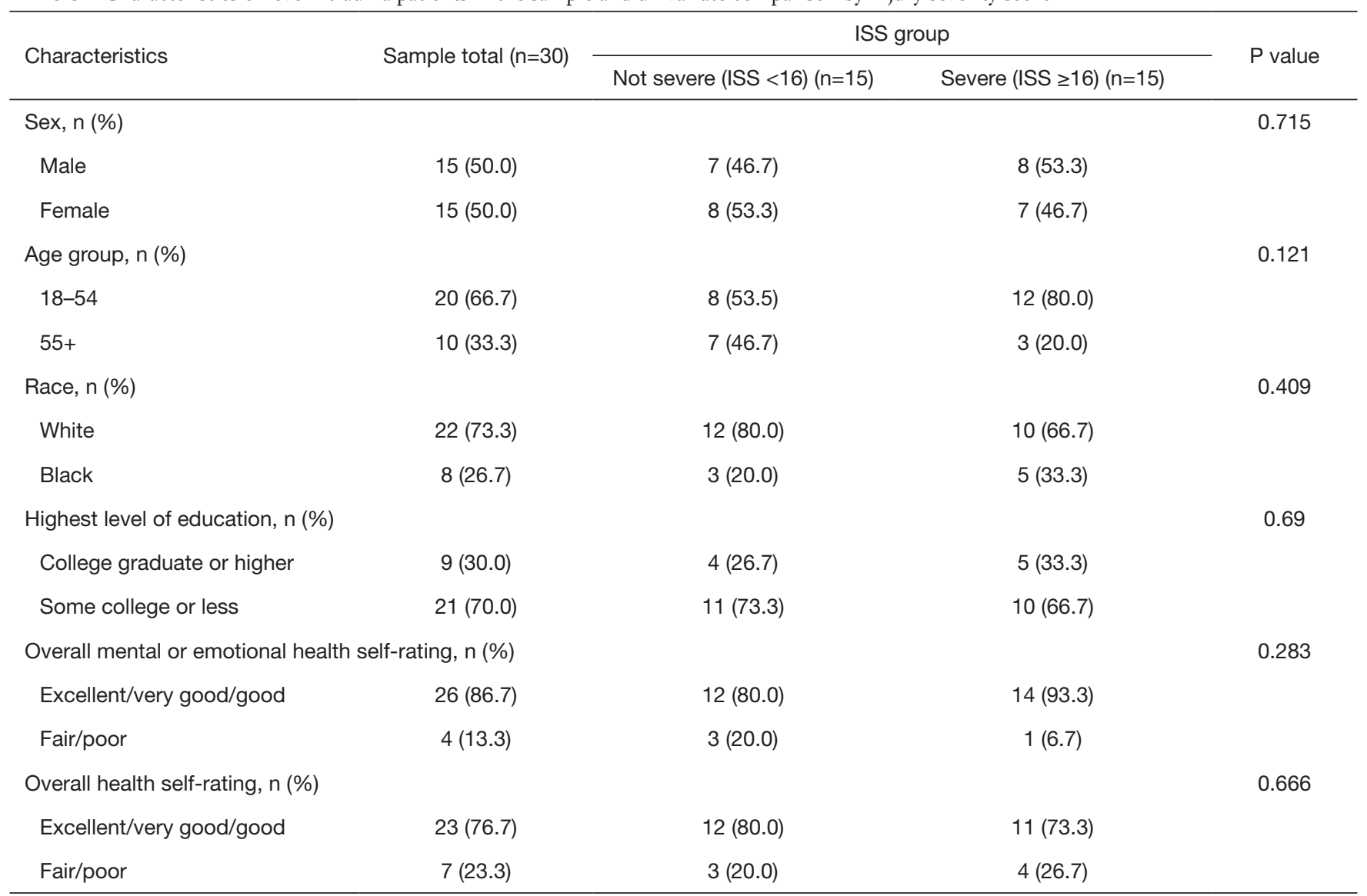

Bivariate comparisons by injury severity group for categorical variables with chi-square test, continuous variables with independent samples $t$-test. ISS, injury severity score.

recommend the hospital to others (57\% decrease, $95 \% \mathrm{CI}$, $-105 \%$ to $-8.6 \%$ ) (Figure 3).

\section{Discussion}

In this cross-sectional study, we have demonstrated the reliability and validity of HCAHPS domains used in this analysis measuring doctor communication, nurse communication, care transition, and global quality scores, specifically to trauma patients at an urban Level I trauma center. Furthermore, a positive patient experience with regards to nurse or doctor communication was significantly associated with lower rates of 30-day readmissions and lower risk of inpatient complications for patients with severe injuries. Under the DM, and supported by this study, positive patient experiences regarding communication have the potential to positively influence patient clinical outcomes. These findings support the importance of continuously enhancing processes related to communication between providers and patients to enhance the intrinsic value of care for trauma patients, optimize value-based reimbursement based on patient experience ratings, and improve health outcomes. To our knowledge, this was the first study to report association between nurse and doctor communications and clinical outcomes for severely injured trauma patients.

Linking payment and public perception to self-reported patient experience encourages continuous improvement of the patient experience to maximize reimbursement and increase demand for services at a specific hospital. To ensure quality and patient experience remain a major focus of health care delivery, the Centers for Medicare \& Medicaid Services (CMS) place significant importance on patient experience self-reports. Under the HVBP, 
Table 2 Comparison of patient satisfaction measures and outcomes by ISS group

\begin{tabular}{|c|c|c|c|c|}
\hline Characteristic & Sample total $(n=30)$ & \multicolumn{2}{|c|}{ ISS group } & $P$ value \\
\hline \multicolumn{5}{|l|}{ Predictors, n (\%) } \\
\hline Positive nurse communication & $15(50.0)$ & $9(60.0)$ & $6(40.0)$ & 0.273 \\
\hline Positive doctor communication & $11(36.7)$ & $6(40.0)$ & $5(33.3)$ & 0.705 \\
\hline Excellent overall health & $3(10.0)$ & $1(6.7)$ & $2(13.3)$ & 0.543 \\
\hline Excellent overall mental health & 7 (23.3) & $2(13.3)$ & 5 (33.3) & 0.195 \\
\hline $\begin{array}{l}\text { Positive would recommend } \\
\text { hospital }\end{array}$ & $24(80.0)$ & $13(86.7)$ & $11(73.3)$ & 0.361 \\
\hline All-cause 30 -day readmission & $7(23.3)$ & $2(13.3)$ & 5 (33.3) & 0.195 \\
\hline $\begin{array}{l}\text { Longer than expected hospital } \\
\text { stay }\end{array}$ & 7 (23.3) & $4(26.7)$ & $3(20.0)$ & 0.516 \\
\hline Hospital-acquired complications & $4(13.3)$ & $1(6.7)$ & $3(20.0)$ & 0.422 \\
\hline Length of stay (mean, SD) & $8.2(6.66)$ & $6.7(4.67)$ & $9.8(8.06)$ & 0.118 \\
\hline
\end{tabular}

Bivariate comparisons by ISS group for categorical variables with chi-square test, continuous variables with Wilcoxon rank test. Nurse communication, doctor communication, and care transition are considered positive when all responses to questions in those categories are all top-box (or the most positive/highest scoring, i.e., "always") responses. Positive "would recommend hospital" indicates the participant selected "definitely yes" to the question asking whether they would recommend the hospital to others. ISS, injury severity score.

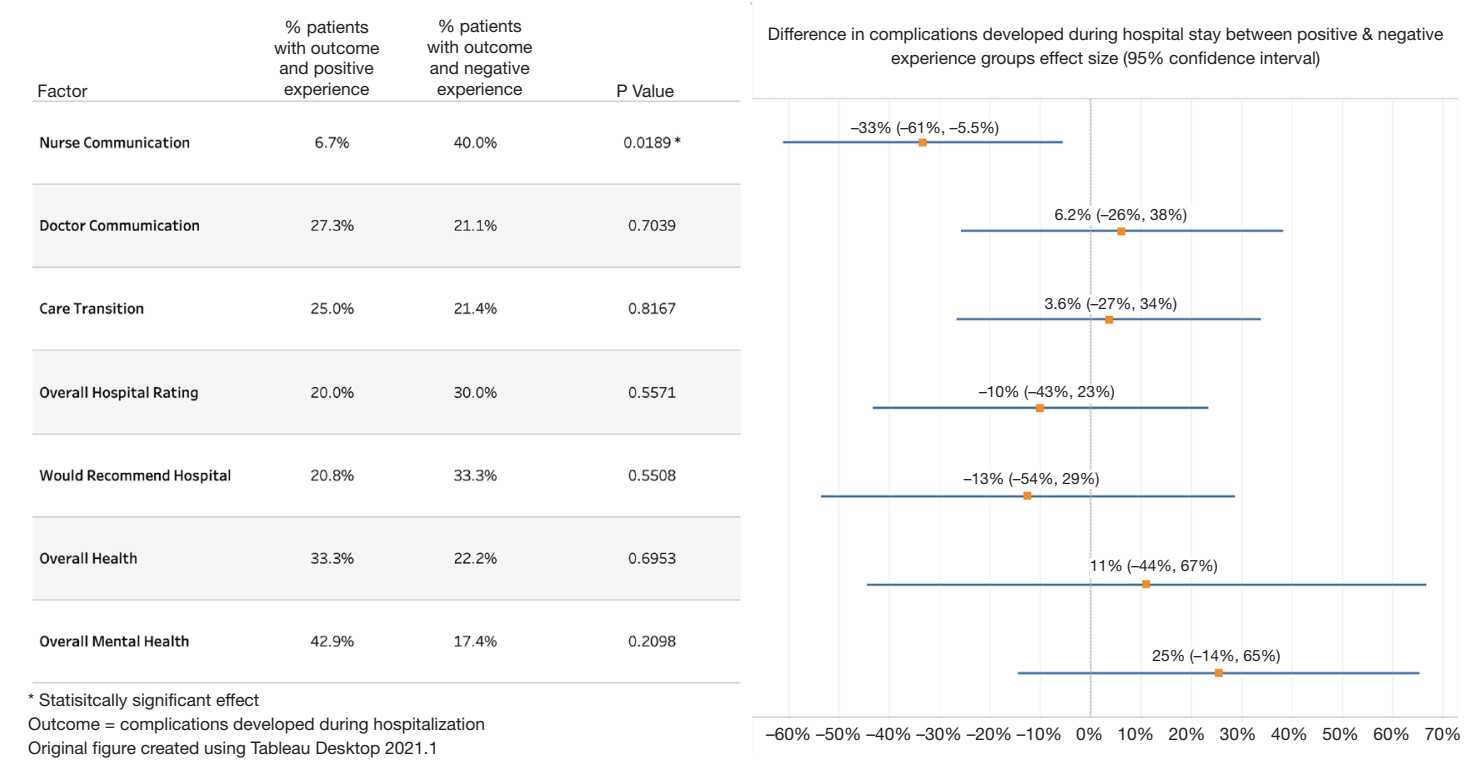

Figure 2 Associations between patient experience and hospital-acquired complications. 


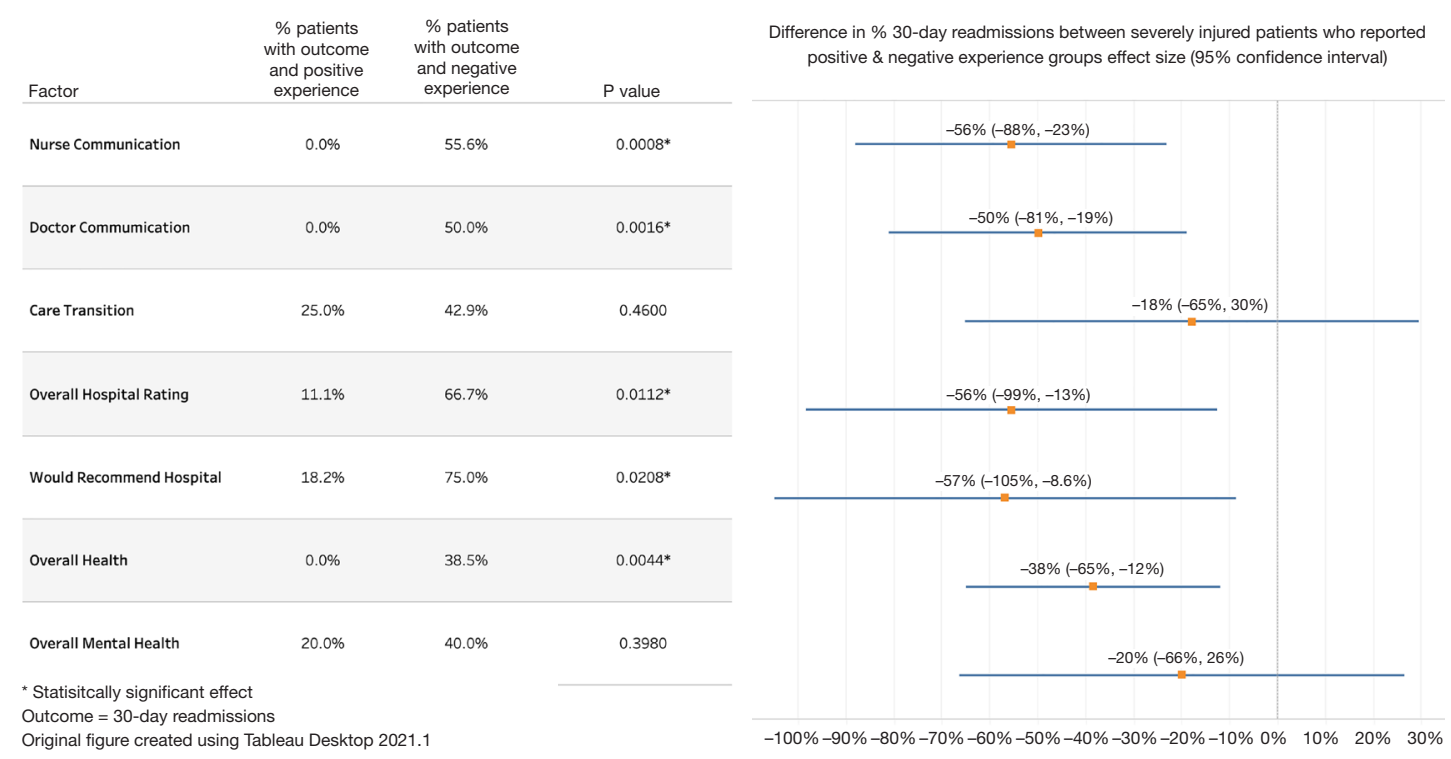

Figure 3 Association between severely injured patient experience (ISS $\geq 16$ ) and 30-day readmissions. ISS, injury severity score.

two percent of Medicare payments reimbursed through the Inpatient Prospective Payment System (IPPS) are redistributed by CMS, and patient experience accounts for $25 \%$ of the performance score that determines these payments $(9,39,44,60)$. Second, the HCAHPS scores are publicly reported, with hospitals being assigned a star rating (one star to five stars) by CMS. Additionally, multiple public reporting systems, such as U.S. News \& World Report Hospital Rankings \& Ratings and Hospital Compare, are integrating patient experience performances into their scoring systems that are available to assist individuals making decisions on where to receive health care services $(9,61)$.

Factors that influence patients' self-reports on hospital experience include expectations patients have regarding their care and recovery, related to preconceived biases towards the health care system, their own health, and previous health care-related experiences (62). For example, a person in good health before being injured may expect their hospitalization to be shorter than it ultimately is. Severe pain, how that pain is managed, and how the patient perceives empathy for pain from providers also have a significantly strong influence on how a patient perceives and reports their experience. Appropriate communication with providers can help patients manage expectations and understand the pain management process $(3,40)$. A patient's experience during discharge is another important series of interactions with care providers because it may ultimately determine whether the patient has an adverse event after hospitalization. Approximately $17 \%$ of people hospitalized are readmitted to acute care within 30 days because inadequate discharge planning and education can make a patient feel uncertain about what to expect during post-acute care and how to respond in certain adverse circumstances (63). Ineffective communication during discharge can also lead an injured patient to be unprepared to self-manage pain at home, which can lead to greater discomfort, distress, and fear (64). Effective discharge planning is crucial because HCAHPS surveys are mailed to a random sample of patients between 48 hours and six weeks after discharge (42). How a patient perceives the cause of an adverse event or the level of preparation during discharge are especially influential on HCAHPS because that individual completes the survey at home while simultaneously being responsible for their own care. Inadequate education during discharge and unsuccessful pain and care management at home will likely negatively impact how a patient rates their experience with the hospital (40).

The importance of nurses' communication with trauma patients in our findings emphasizes the strategic benefit of investing in continuous improvement of structures and processes that support nursing communication. A systematic review conducted by Rouleau and colleagues (65) found 22 articles that report the efficacy of a nursing care performance framework (NCPF), based partially on the 
DM, for describing the improvement of nursing-sensitive patient conditions (outcomes) by supporting nurses with the necessary resources (structure: staff supply, working conditions, economic stability) that strengthen nursing services (processes: assessment, care planning, evaluation).

There are several limitations to our study that should be considered when interpreting results. First, although our study's response rate of $31.5 \%$ is greater than the response rate achieved in the national HCAHPS survey (26.7\% in 2017) (66), the major limitation of this study is the small sample size of 30 participants. With only 30 participants, we were not able to apply multivariate models to adjust for potential confounders or attempt to prove causal connections. This small sample size is due to having a small potential sample size of only 95 patients. These 95 individuals participated in a previous research study in the site hospital, which consisted of 225 patients, and consented to being contacted later for future research. This small sample size caused some of our statistical tests to not meet the criteria for appropriately comparing groups. Independent samples $t$-tests to compare the means of two groups using continuous variables (ISS, LOS) should have a sample size of at least 30 and each cell of a chi-square test of homogeneity for categorical variables (HACs, readmissions) should have a count of at least five (67), but several cells in our chi-square tests had counts of less than five. Furthermore, we believe the small sample size led to an upper CI interval greater than $100 \%$ in the "would recommend hospital" in analysis for the severely injured subgroup. Although this major limitation impacts the validity of the resulting relationships between patient experience and outcomes, this study presents an exploratory perspective on the topic and informs the development of further research that can more definitively test the proposed hypothesis. Future research should include a larger sample size that will allow analysis of causal relationships, provide more context to why patients have and perceive different experiences, reduce biases, and improve internal validity. Second, a cross-sectional study design restricts the ability to adjust for nonresponse bias, as patients with extreme positive or extreme negative experiences are more likely to respond to a survey (68). Even though the HCAHPS survey has been thoroughly tested in the U.S., our study is still subject to uncertainties about those who did not respond. In response to this limitation, the study sample and non-responders were compared in key demographic and health related factors (Table 3). There were 95 total potential patients. Thirty participated in the study and 65 did not. These two groups were statistically significantly different in mean age $(\mathrm{P}=0.04)$, ISS $(\mathrm{P}=0.002)$, and LOS $(\mathrm{P}=0.008)$. The two groups, however, did not have a statistically significant difference in proportions in sex or race. Participants were significantly older, more severely injured, and had longer lengths of stay than patients who did not respond to the survey but differences in proportions were not significant for gender $(\mathrm{P}=0.133)$ and race $(\mathrm{P}=0.053)$. Given study participants and nonresponders differed significantly in health-related factors, which are known to influence HCAHPS scores (62), it is possible experience scores would have been different if the sample size was larger and differences in health factors between participants and non-responders were not significant. Third, the clinical outcomes were selfreported and were subject to recall-bias. The standard cutoff time for HCAHPS responses is six weeks, but the average time between hospital discharge and study survey was almost one year. We were able to manage recall bias for several of the survey questions related to health factors, such as readmissions and complications, by confirming these responses in the participants' EHRs. While all these responses were recalled correctly by participants, we were unable to perform similar confirmations on responses about their interactions with care providers. However, Black and Jenkinson (69) state that a delay between care delivery and a survey asking about that experience and associated outcomes may be needed to allow the patient to realize all potential benefits of their health care experience and distinguish between minor inconveniences and serious failings by providers. Associations between experience and clinical outcomes are not causal relationships, which limits the claim that improving patient experience will lead to better health outcomes. Furthermore, these associations could be confounded by other differences in the patients. Future research that reduces time between discharge and survey, and includes additional patient characteristics, such as insurance status or comorbidities, would allow more complete analysis of causal relationships to provide greater context into patient experience while controlling for a larger number of important patient factors. For example, a global study found that individuals with high morbidity were more likely to report poor patient experience that those with low morbidity (70). Interviewer bias is another limitation inherent in a phone-based survey study. The differing ways multiple interviewers may phrase or order 
Table 3 Comparison of study participants and non-responders

\begin{tabular}{|c|c|c|c|c|}
\hline Characteristic & Sample total $(\mathrm{N}=95)$ & \multicolumn{2}{|c|}{ Survey respondent group } & $P$ value \\
\hline Age (mean, SD) & $49.6(17.81)$ & $55.1(17.56)$ & $47.1(17.48)$ & $0.04^{*}$ \\
\hline ISS (mean, SD) & $13.0(7.48)$ & $16.5(8.91)$ & $11.5(6.18)$ & $0.002^{*}$ \\
\hline LOS (mean, SD) & $6.2(5.19)$ & $8.2(6.66)$ & $5.3(4.07)$ & $0.008^{*}$ \\
\hline Female & 37 (38.9) & $15(40.5)$ & $22(59.5)$ & \\
\hline Male & $58(61.1)$ & $15(25.9)$ & $43(74.1)$ & \\
\hline Race (N, \%) & & & & 0.053 \\
\hline Black & $39(41.1)$ & $8(20.5)$ & $31(79.5)$ & \\
\hline
\end{tabular}

Bivariate comparisons by responder group for categorical variables with chi-square test, continuous variables with independent samples $t$-test. *, statistically significant difference. SD, standard deviation; ISS, injury severity score; LOS, length of stay.

questions in a survey can influence the way participants respond and how data are collected (71). To reduce interviewer bias, a phone script including exact phrasing for each survey question and set of possible responses was developed by the authors and strictly followed for each phone call. Taking the outlined limitations into account, our study provided new exploratory evidence about the importance of patient experience, especially in the form of communication with providers, for hospitalized trauma patient outcomes.

\section{Conclusions}

In this cross-sectional study, we were able to gather 30 responses from 95 discharged trauma patients through phone interviews. The reliability and validity of these surveys were tested using correlation tests. Overall, positive nurse communications were associated with 33\% fewer complications during hospital stays. Furthermore, we observed more significant associations with patient experience measures for patients with severe injuries, with positive communication reported between patients and providers being associated with lower risk of 30-day readmissions. These results support the continuous focus on studying and improving patient experience for all trauma patients, especially for those with more severe injuries, and bolstering the role of communication in enhancing the experience of trauma patients. The importance of patient experience in hospital payment and reputation suggests that trauma departments should focus on continuously improving patient experience processes, especially in the domains of communication, and that nurses and doctors should be leaders in these efforts. Doing so may allow hospitals to capitalize on the benefits of value-based payment systems and ensure trauma patients receive the highest quality care and best outcomes possible.

\section{Acknowledgments}

We would like to thank Dr. Enbal Shacham for her mentorship in primary data collection study design. We would also like to thank all the participants involved in the study for sharing their experiences despite the struggle overcoming traumatic injury can sometimes be... you were the inspiration for this study.

Funding: None.

\section{Footnote}

Provenance and Peer Review: This article was commissioned by the Guest Editors (Stephen J. O'Connor, Nancy Borkowski and Katherine A. Meese) for the series "Shaping Tomorrow's Healthcare Systems: Key Stakeholders' Expectations and Experiences" published in Fournal of Hospital Management and Health Policy. The article has undergone external peer review. 
Reporting Checklist: The authors have completed the STROBE reporting checklist. Available at https://jhmhp. amegroups.com/article/view/10.21037/jhmhp-21-65/rc

Data Sharing Statement: Available at https://jhmhp. amegroups.com/article/view/10.21037/jhmhp-21-65/dss

Peer Review File: Available at https://jhmhp.amegroups.com/ article/view/10.21037/jhmhp-21-65/prf

Conflicts of Interest: All authors have completed the ICMJE uniform disclosure form (available at https://jhmhp. amegroups.com/article/view/10.21037/jhmhp-21-65/coif). The series "Shaping Tomorrow's Healthcare Systems: Key Stakeholders' Expectations and Experiences" was commissioned by the editorial office without any funding or sponsorship. AO works with trauma patients in the study's site hospital as a peer support visitor and helps with the hospital's support program development in his role at the academic medical center as an employee of the School of Medicine. VM is employed as the site hospital's trauma research coordinator. $\mathrm{AO}$ and $\mathrm{VM}$ received a grant $(\$ 10,823)$ from the Society of Trauma Nurses in January 2018 to conduct an unrelated trauma study that contributed to the identification and recruitment of patients eligible for this study. The authors have no other conflicts of interest to declare.

Ethical Statement: The authors are accountable for all aspects of the work in ensuring that questions related to the accuracy or integrity of any part of the work are appropriately investigated and resolved. The study was conducted in accordance with the Declaration of Helsinki (as revised in 2013). The study was approved by the Saint Louis University Institutional Review Board (IRB No. 31075) and informed consent was given by all individuals before participating in the survey.

Open Access Statement: This is an Open Access article distributed in accordance with the Creative Commons Attribution-NonCommercial-NoDerivs 4.0 International License (CC BY-NC-ND 4.0), which permits the noncommercial replication and distribution of the article with the strict proviso that no changes or edits are made and the original work is properly cited (including links to both the formal publication through the relevant DOI and the license). See: https://creativecommons.org/licenses/by-nc-nd/4.0/.

\section{References}

1. GBD 2019 Diseases and Injuries Collaborators. Global burden of 369 diseases and injuries in 204 countries and territories, 1990-2019: a systematic analysis for the Global Burden of Disease Study 2019. Lancet 2020;396:1204-22.

2. Haagsma JA, Graetz N, Bolliger I, et al. The global burden of injury: incidence, mortality, disability-adjusted life years and time trends from the Global Burden of Disease study 2013. Inj Prev 2016;22:3-18.

3. Archer KR, Abraham CM, Song Y, et al. Cognitivebehavioral determinants of pain and disability two years after traumatic injury: A cross-sectional survey study. J Trauma Acute Care Surg 2012;72:473-9.

4. Dietch Z, Young JS, Young SD. Analysis of Profit and Loss by Injury Severity over 20 Years at a University Level I Trauma Center. Am Surg 2016;82:644-8.

5. Silvester L. Major trauma: a Lean approach to process design. British Journal of Healthcare Management 2011;17:11.

6. Donabedian A. The quality of care. How can it be assessed? JAMA 1988;260:1743-8.

7. Moore L, Lavoie A, Bourgeois G, et al. Donabedian's structure-process-outcome quality of care model: Validation in an integrated trauma system. J Trauma Acute Care Surg 2015;78:1168-75.

8. Moore L, Stelfox HT, Turgeon AF, et al. Rates, patterns, and determinants of unplanned readmission after traumatic injury: a multicenter cohort study. Ann Surg 2014;259:374-80.

9. Joseph B, Azim A, O'Keeffe T, et al. American College of Surgeons Level I trauma centers outcomes do not correlate with patients' perception of hospital experience. J Trauma Acute Care Surg 2017;82:722-7.

10. Manary MP, Boulding W, Staelin R, et al. The patient experience and health outcomes. N Engl J Med 2013;368:201-3.

11. Tsai TC, Orav EJ, Jha AK. Patient satisfaction and quality of surgical care in US hospitals. Ann Surg 2015;261:2-8.

12. Rathert C, Wyrwich MD, Boren SA. Patient-centered care and outcomes: a systematic review of the literature. Med Care Res Rev 2013;70:351-79.

13. Santana MJ, Manalili K, Jolley RJ, et al. How to practice person-centred care: A conceptual framework. Health Expect 2018;21:429-40.

14. White B, Driver S, Warren AM. Resilience and indicators of adjustment during rehabilitation from a spinal cord injury. Rehabil Psychol 2010;55:23-32. 
15. DiMaggio C, Ayoung-Chee P, Shinseki M, et al. Traumatic injury in the United States: In-patient epidemiology 20002011. Injury 2016;47:1393-403.

16. Murray CJ, Vos T, Lozano R, et al. Disability-adjusted life years (DALYs) for 291 diseases and injuries in 21 regions, 1990-2010: a systematic analysis for the Global Burden of Disease Study 2010. Lancet 2012;380:2197-223.

17. Prevention CfDCa. Cost of Injury Reports, Data \& Statistics (WISQARS)-Nonfatal Injury Inquiry Queries. 2019. Available online: https://www.cms.gov/Medicare/ Quality-Initiatives-Patient-Assessment-Instruments/ HospitalQualityInits/HospitalHCAHPS (Accessed 31 August 2020).

18. Institute NT. Trauma Statistics. 2014. Available online: https://www.nattrauma.org/trauma-statistics-facts/ (Accessed 16 March 2017).

19. Quality AfHCRa. What is Patient Experience? 2021. Available online: https://www.ahrq.gov/cahps/about-cahps/ patient-experience/index.html (2021).

20. Jenkinson C, Coulter A, Bruster S. The Picker Patient Experience Questionnaire: development and validation using data from in-patient surveys in five countries. Int $\mathrm{J}$ Qual Health Care 2002;14:353-8.

21. Grosios K, Gahan PB, Burbidge J. Overview of healthcare in the UK. EPMA J 2010;1:529-34.

22. Mackie P. Shifting the balance of power in the NHS. Health and Hygiene 2002;23:4-5. Available online: http:// explore.bl.uk/primo_library/libweb/action/display.do?ta $\mathrm{bs}=$ details $\mathrm{Tab} \&$ gathStat $\mathrm{Tab}=$ true $\& \mathrm{ct}=$ display $\& \mathrm{fn}=$ searc h\&doc=ETOCRN111214451\&indx=1\&recIds=ETOC RN111214451 (Accessed 27 Jan 2020).

23. Coulter A, Locock L, Ziebland S, et al. Collecting data on patient experience is not enough: they must be used to improve care. BMJ 2014;348:g2225.

24. Doyle C, Lennox L, Bell D. A systematic review of evidence on the links between patient experience and clinical safety and effectiveness. BMJ Open 2013;3:001570.

25. Arah OA, ten Asbroek AHA, Delnoij DMJ, et al. Psychometric properties of the Dutch version of the hospital-level consumer assessment of health plans survey instrument. Health Serv Res 2006;41:284-301.

26. Smirnova A, Lombarts KMJMH, Arah OA, et al. Closing the patient experience chasm: A two-level validation of the Consumer Quality Index Inpatient Hospital Care. Health Expect 2017;20:1041-8.

27. Auras S, de Cruppe W, Blum K, et al. Mandatory quality reports in Germany from the hospitals' point of view: a cross-sectional observational study. BMC Health Serv Res 2012;12:378.

28. Kraska RA, Weigand M, Geraedts M. Associations between hospital characteristics and patient satisfaction in Germany. Health Expect 2017;20:593-600.

29. Schoenfelder T, Klewer J, Kugler J. Determinants of patient satisfaction: a study among 39 hospitals in an in-patient setting in Germany. Int J Qual Health Care 2011;23:503-9.

30. Mainz J, Kristensen S, Bartels P. Quality improvement and accountability in the Danish health care system. Int J Qual Health Care 2015;27:523-7.

31. Garratt A, Solheim E, Danielsen K. National and CrossNational Surveys of Patient Experiences: A Structured Review. NIPH Methods Resources. Oslo, Norway 2008.

32. OECD Health Ministerial Statement - The next generation of health reforms. 201701/18.

33. Schoen C, Osborn R, How SK, et al. In chronic condition: experiences of patients with complex health care needs, in eight countries, 2008. Health Aff (Millwood) 2009;28:w1-16.

34. Berwick DM. A user's manual for the IOM's 'Quality Chasm' report. Health Aff (Millwood) 2002;21:80-90.

35. Institute of Medicine Committee on Quality of Health Care in A. Crossing the Quality Chasm: A New Health System for the 21st Century. Washington (DC): National Academies Press (US). Copyright 2001 by the National Academy of Sciences. All rights reserved.2001.

36. HCAHPS. HCAHPS Summary Analysis. 2019. Available online: https://www.hcahpsonline.org/en/summaryanalyses/ (Accessed 20 Sept 2019).

37. Shulman BS, Crowe B, Hutzler L, et al. Socioeconomic Status Negatively Affects HCAHPS Scores in Orthopedic Patients The Results of 15,789 Patients at a Single Institution. Bull Hosp Jt Dis (2013) 2018;76:216-20.

38. Blumenthal D, Abrams M, Nuzum R. The Affordable Care Act at 5 Years. N Engl J Med 2015;373:1580.

39. Chee TT, Ryan AM, Wasfy JH, et al. Current State of Value-Based Purchasing Programs. Circulation 2016;133:2197-205.

40. Otani K, Chumbler NR, Herrmann PA, et al. Impact of Pain on Patient Satisfaction Integration Process: How Patients With Pain Combine Their Health Care Attribute Reactions. Health Serv Res Manag Epidemiol 2015;2:2333392815615103.

41. O'Malley AJ, Zaslavsky AM, Hays RD, et al. Exploratory 
factor analyses of the CAHPS Hospital Pilot Survey responses across and within medical, surgical, and obstetric services. Health Serv Res 2005;40:2078-95.

42. Services CfMaM. Hospital Compare. 2019. Available online: https://www.medicare.gov/hospitalcompare/search. html? (Accessed 27 Jan 2020).

43. Elliott MN, Cohea CW, Lehrman WG, et al. Accelerating Improvement and Narrowing Gaps: Trends in Patients' Experiences with Hospital Care Reflected in HCAHPS Public Reporting. Health Serv Res 2015;50:1850-67.

44. VanLare JM, Conway PH. Value-based purchasing-national programs to move from volume to value. N Engl J Med 2012;367:292-5.

45. Lake ET, Germack HD, Viscardi MK. Missed nursing care is linked to patient satisfaction: a cross-sectional study of US hospitals. BMJ Qual Saf 2016;25:535-43.

46. Orindi BO, Lesaffre E, Quintero A, et al. Contribution of HCAHPS Specific Care Experiences to Global Ratings Varies Across 7 Countries: What Can be Learned for Reporting These Global Ratings? Med Care 2019;57:e65-72.

47. Moore L, Stelfox HT, Evans D, et al. Trends in Injury Outcomes Across Canadian Trauma Systems. JAMA Surg 2017;152:168-74.

48. Parreco J, Buicko J, Cortolillo N, et al. Risk factors and costs associated with nationwide nonelective readmission after trauma. J Trauma Acute Care Surg 2017;83:126-34.

49. Surgeons ACo. National Trauma Data Bank 2016: Annual Report 2016.

50. West SK, O'Mara MS, Spalding MC. Using clinical analysis to classify 30-day trauma readmissions. J Trauma Acute Care Surg 2018;85:613-9.

51. Steel DG, Holt D. Analysing and Adjusting Aggregation Effects: The Ecological Fallacy Revisited. International Statistical Review 1996;64:22.

52. Kahn SA, Iannuzzi JC, Stassen NA, et al. Measuring satisfaction: factors that drive hospital consumer assessment of healthcare providers and systems survey responses in a trauma and acute care surgery population. Am Surg 2015;81:537-43.

53. Palmer C. Major trauma and the injury severity score-where should we set the bar? Annu Proc Assoc Adv Automot Med 2007;51:13-29.

54. Keller S, O'Malley AJ, Hays RD, et al. Methods used to streamline the CAHPS Hospital Survey. Health Serv Res 2005;40:2057-77.

55. Tavakol M, Dennick R. Making sense of Cronbach's alpha.
Int J Med Educ 2011;2:53-5.

56. Hu Y, Zhang Z, Xie J, et al. The Outpatient Experience Questionnaire of comprehensive public hospital in China: development, validity and reliability. Int J Qual Health Care 2017;29:40-6.

57. Thiels CA, Hanson KT, Yost KJ, et al. Achieving a 5-star rating: Analysis of Hospital Consumer Assessment of Healthcare Providers and Systems (HCAHPS) scores among patients undergoing elective colorectal operations. Surgery 2016;160:902-14.

58. Aiken LH, Clarke SP, Cheung RB, et al. Educational levels of hospital nurses and surgical patient mortality. JAMA 2003;290:1617-23.

59. SAS Institute Inc. SAS/ACCESS ® 9.4, 2013.

60. Joynt KE, Rosenthal MB. Hospital value-based purchasing: will Medicare's new policy exacerbate disparities? Circ Cardiovasc Qual Outcomes 2012;5:148-9.

61. Austin JM, Jha AK, Romano PS, et al. National hospital ratings systems share few common scores and may generate confusion instead of clarity. Health Aff (Millwood) 2015;34:423-30.

62. Zhang Y, Rohrer J, Borders T, et al. Patient satisfaction, self-rated health status, and health confidence: an assessment of the utility of single-item questions. Am J Med Qual 2007;22:42-9.

63. Graumlich JF, Novotny NL, Aldag JC. Brief scale measuring patient preparedness for hospital discharge to home: Psychometric properties. J Hosp Med 2008;3:446-54.

64. Goldsmith H, McCloughen A, Curtis K. Using the trauma patient experience and evaluation of hospital discharge practices to inform practice change: A mixed methods study. J Clin Nurs 2018;27:1589-98.

65. Rouleau G, Gagnon MP, Côté J, et al. Impact of Information and Communication Technologies on Nursing Care: Results of an Overview of Systematic Reviews. J Med Internet Res 2017;19:e122.

66. Godden E, Paseka A, Gnida J, et al. The impact of response rate on Hospital Consumer Assessment of Healthcare Providers and System (HCAHPS) dimension scores. Patient Experience Journal 2019;6:9.

67. Triola MM, Triola MF, Roy J. Biostatistics for the Biological and Health Sciences: Biost Biolo Healt PDF_2d _2. Madison: Pearson Education, 2017.

68. Perneger TV, Chamot E, Bovier PA. Nonresponse bias in a survey of patient perceptions of hospital care. Med Care 2005;43:374-80. 
69. Black N, Jenkinson C. Measuring patients' experiences and outcomes. BMJ 2009;339:b2495.

70. Burgers JS, Voerman GE, Grol R, et al. Quality and coordination of care for patients with multiple conditions: results from an international survey of patient experience.

doi: $10.21037 /$ jhmhp-21-65

Cite this article as: Oberle A, Tian D, Hayes K, Howard S, Moran V. Associations between patient experience and clinical outcomes at a level I trauma center: a cross-sectional surveybased study. J Hosp Manag Health Policy 2022;6:25.
Eval Health Prof 2010;33:343-64.

71. Jager KJ, Tripepi G, Chesnaye NC, et al. Where to look for the most frequent biases? Nephrology (Carlton) 2020;25:435-41. 


\section{Supplementary}

\section{Appendix 1: survey questions}

Please answer the following questions as they refer to your hospitalization after your traumatic injury.

- During this hospital stay, how often did nurses treat you with courtesy and respect?

Never/sometimes/usually/always

- During this hospital stay, how often did nurses listen carefully to you?

Never/sometimes/usually/always

- During this hospital stay, how often did nurses explain things in a way you could understand?

Never/sometimes/usually/always

- During this hospital stay, how often did doctors treat you with courtesy and respect?

Never/sometimes/usually/always

- $\quad$ During this hospital stay, how often did doctors listen carefully to you?

Never/sometimes/usually/always

- During this hospital stay, how often did doctors explain things in a way you could understand?

Never/sometimes/usually/always

- Using any number from 0 to 10 , where 0 is the worst hospital possible and 10 is the best hospital possible, what number would you use to rate this hospital during your stay?

Worst hospital possible $=0 \rightarrow$ best hospital possible $=10$

- Would you recommend this hospital to your friends and family?

Definitely no/probably no/probably yes/definitely yes

- During this hospital stay, staff took my preferences and those of my family or caregiver into account in deciding what my health care needs would be when I left.

Strongly disagree/disagree/agree/strongly agree

- When I left the hospital, I had a good understanding of the things I was responsible for in managing my health.

Strongly disagree/disagree/agree/strongly agree

- When I left the hospital, I clearly understood the purpose for taking each of my medications.

Strongly disagree/disagree/agree/strongly agree

- After discharged from this hospital stay, did you have inpatient visit or emergency room visit within 30 days for any reason?

None/Inpatient visit only/emergency room visit only/both inpatient and emergency room visit

- During this hospital stay, did you have to stay in the hospital longer than you expected?

Yes/no/not sure

- $\quad$ During this hospital stay, did you have any of the following?

Pneumonia/urinary tract infection/drug and alcohol withdrawal syndrome/thrombophlebitis/unplanned intubation/ cardiac arrest/unplanned return to ICU/acute kidney injury/none of the above/not sure

Please answer the following questions about yourself.

- What is your age?

$$
18-24 / 25-34 / 35-44 / 45-54 / 55-64 / 65-74 / 75-84 / 85+
$$

- What is your sex?

Male/female

- Choose one or more races that you consider yourself to be:

White/Black or African American/American Indian or Native American/Asian/Native Hawaiian or Pacific Islander/ other

- What is the highest level of grade or level of school that you have completed?

$8^{\text {th }}$ grade or less/some high school but did not graduate/high school graduate or GED/some college or 2-year degree/4- 
year college graduate/more than 4-year college degree

- What is your zipcode?

- In general, how do you rate your overall health?

Excellent/very good/good/fair/poor

- In general, how would you rate your overall mental or emotional health? Excellent/very good/good/fair/poor 